\title{
GPPS-CH-2020-4
}

\section{DEFICITS IN RANS MODELLING IN THE U-TURN OF A MULTISTAGE CENTRIFUGAL COMPRESSOR}

\author{
Bastian Dolle \\ University of Duisburg Essen \\ bastian.dolle@uni-due.de \\ Duisburg, Germany
}

\author{
Viktor Hermes \\ Siemens Gas and Power \\ GmbH \& Co. KG \\ Duisburg, Germany
}

\author{
Dieter Brillert \\ University of Duisburg Essen \\ Duisburg, Germany
}

\begin{abstract}
Multi-stage radial compressors are key components in many industrial applications. Today, downsizing the compressor to reduce capital expenditure (CAPEX) is of utmost importance. Accordingly, the request is to increase the pressure ratio in a most compact design, accepting nearly no penalties in the performance level.

Here, the complex flow in stator parts of multistage centrifugal compressors was investigated and predictions from numerical simulations were compared with experimental data. A test rig was developed enabling steady-state data using five-hole-probes and time-resolved velocity data via hot-film-anemometry to be acquired. Reynolds-averaged Navier-Stokes (RANS) calculations showed significant deviations from the measured data in predicting the flow profiles in the return bend for high flow coefficients $(\varphi \geq 0.12)$ were observed. Not only eddy-viscosity models but also Reynolds stress models show similar discrepancies. Large Eddy Simulation (LES) was applied to acquire more detailed information about the flow. The flow profiles predicted by a hybrid RANS-LES calculation show better agreement with the experimental data but performance data still vary. Concluding, the RANS approach was found to under-predict turbulent transport in highly curved and complex geometries at high flow coefficients. This fact must be understood and respected when using CFD-techniques for flow calculation in wide return channels of centrifugal compressors.
\end{abstract}

\section{INTRODUCTION}

Multistage centrifugal compressors, used in a wide range of industrial applications, are often designed in a single shaft configuration. Each single compressor stage comprises a rotating impeller and a non-rotating diffuser and return channel. The need to lead the radial outward impeller flow back and then axially on to the next impeller inlet results in a challenging geometry design. This typically consists of a vaned or vaneless radial diffuser, a highly curved return bend, a vaned return channel and finally a $90^{\circ}$-bend. Simultaneously, the swirl induced by the preceding impeller must be eliminated completely before the next stage is entered. Key aims in optimizing the stator are to achieve a homogeneous and swirl-free outflow while minimizing total pressure losses and maximizing the increase of static pressure. Pursuing these goals is more challenging at off-design and especially under deep part load conditions.

As the flow path is complex, the accessibility within return channels for measurement applications such as flow probes or optical measurement techniques is limited. Nevertheless, several experimental studies have led to a better understanding of flow behavior and thereby to improved design guidelines. To investigate stator flow, and in particular, return channels, Rothstein [1] tested stator geometries with different flow coefficients focusing on comparatively low flow rates and consequent narrow channel widths. He concluded that using a return vane with high curvature in the first part and low curvature in the last part to straighten the flow will result in a better exit flow profile and better performance. Lenke [2] subsequently applied an algebraic Reynolds Stress model and simulated the flow in Rothstein's geometries. His findings support Rothstein`s conclusions. Recent publications by Aalburg et al. [3] and Rossbach ([4], [5]) focus on detailed flow phenomena with the aim of reducing the diffuser radius ratio, in turn motivated by the manufacturer need to reduce CAPEX by reducing machine size. However, with a lower diffuser ratio, the flow velocity inside the return bend and the return guide vanes (RGV) increases, promoting secondary flows and thus increased turbulence intensity due to higher shear rates.

Numerical modelling in these contexts presents a particular challenge: modern turbulence models, such as 
eddy viscosity models, exhibit deficits in predicting turbulent effects in flows with curved walls, secondary flows, rotating flow and in three-dimensional flows in general as summarized by Wilcox [6]. All such flow types are present in return channels and become intensified when its' outer diameters are reduced.

In addition, reducing the diffuser ratio relies in reducing the return vane radial length, which leads to higher blade loading and thus to swirl remaining at the stage outlet, shown numerically by Bisping et al. [7] showed numerically. To reduce blade loading and the outlet flow angle, the vane leading edge might be shifted further upstream in the $180^{\circ}$-bend. Various vane designs have been studied. Veress and Van den Braembussche [8] improved the stage performance with an RGV leading edge starting half way along the length of the radial diffuser. However, a pair of vortices developed inside the vaned $180^{\circ}$-bend, their intensity could be reduced with a negative lean angle. Other designs use leading edges starting at the bend inlet like Aalburg et al. [9] did. de Bellis et al. [10] investigated a return channel with a return guide vane starting in the bend zenith. Jariwala et al. [11] demonstrate a promising reduction and homogenization of the outlet flow angle with a leading edge starting centered in the second half of the return bend. The manufacturing effort of this particular design is a good compromise between simple cylindrical vanes and long three-dimensional vanes starting in the diffuser.

The design challenge presented by so-called elbow or boomerang vanes is that the number of design parameters increases due to the three-dimensional leading edge or vane lean angles [8]. Parameter optimization therefore only becomes feasible with reliable computational fluid dynamic (CFD) calculations. Unfortunately, even modern, highly sophisticated CFD-codes exhibit uncertainties in predicting flow, especially in complex flow paths.

Our previous research [12] has investigated differences between experimental and numerical data in this context, with findings supporting those of Kim and Chen [13]. RANS turbulence models were shown to be unable to give precise predictions of the flow profiles in return bends for high flow coefficients; specifically, such models tend to underpredict turbulent effects in flow areas with vanished velocity gradients but high velocity levels. Thus, lateral turbulent transport is suppressed and high-energetic flow regions are conserved.

Only a few literature resources discuss but illustrate that RANS models are not suitable for return channel optimizations at high flow coefficients. Therefore, additional research on improving RANS models is necessary. Thus, an advanced hybrid URANS/LES method in addition with time-resolved experimental velocity data is used in this paper to amplify knowledge on turbulence in stator flows and on deficits in turbulence modelling.

\section{TEST RIG}

Shown, experimental data is acquired using a stator test rig comprising a radial diffuser, return bend, RGV and a subsequent $90^{\circ}$-turn, as shown in Fig. 1.

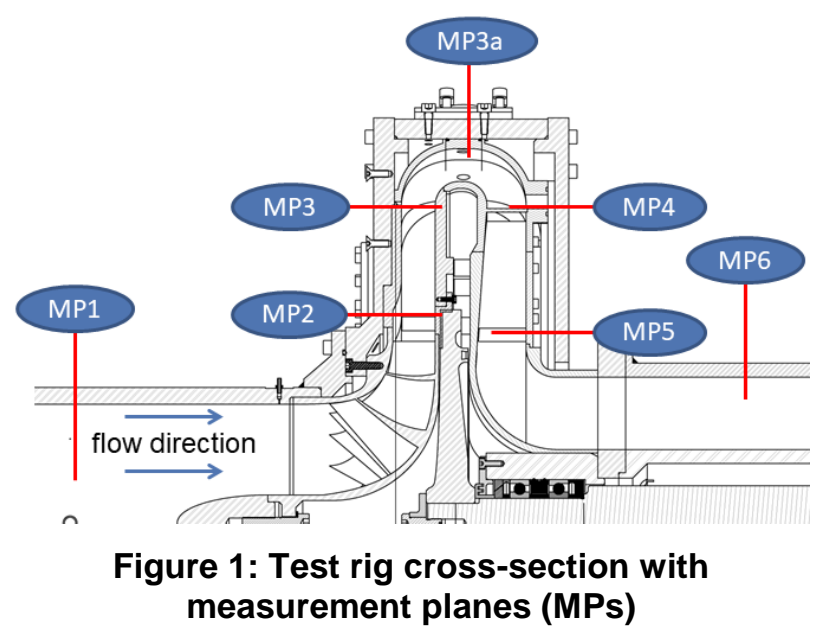

Air, as a working fluid, is provided by a 4-stage axial compressor because a non-rotating pre-swirler upstream to the test section is used instead of an impeller. For each operating point, a pre-swirler is designed to match the impeller outlet flow regarding velocity and flow angle profiles. The test rig is equipped with pressure tabs and flush mount pressure transducers in each measuring plane (Fig. 1) to measure static and dynamic pressure. More detailed information on the test rig can be found in [12].

A number of measurement planes allow access for five-hole probes and hot-film anemometry, enabling complete steady-state and time-resolved velocity profiles to be captured. Stagnation and static pressure profiles are mass-weight averaged to calculate pressure loss and pressure recovery coefficients according to

$$
\begin{gathered}
C_{p}=\frac{p_{o u t}-p_{\text {in }}}{p_{t, \text { in }}-p_{\text {in }}} \\
\zeta=\frac{p_{t, o u t}-p_{t, \text { in }}}{p_{t, \text { in }}-p_{\text {in }}}
\end{gathered}
$$

All spanwise flow profiles shown are area averaged in the circumferential direction and normalized with the equivalent averaged value at the diffuser outlet (MP3).

\section{NUMERICAL METHODS}

For industrial purposes, three-dimensional numerical simulations are only economical when using the eddy viscosity approach to turbulence modelling. To close this approach, the Menter two-equation Shear Stress Transport model (SST) [14] based on the $k-\omega$ model is widely used for stability reasons and its low computational costs. The SST model is applied in this study to represent eddy viscosity models. To improve numerical results to accord better with experimental data, especially in separated flows [12], the "reattachment 
modification" is available in ANSYS CFX which is an additional production term in the k-equation. The SST reattachment modification results in an increase in turbulent kinetic energy in regimes with highly free shear layers to expedite reattachment. Due to the isotropic character of eddy viscosity turbulence models, directional differences in turbulence cannot be considered. Reynolds stress (RS) models, in contrast, are capable of respecting anisotropic effects in turbulent flows; however, this accuracy benefit comes with increased computational demand as there are five additional equations to solve. The symmetric Reynolds stress tensor is modelled with six equations and closed with a seventh equation for the turbulent dissipation rate $\varepsilon$. This set of equations also leads to stability problems in many flows, which is why industrial use of RS models is limited. In this contribution, the SSG model [15] is used as a Reynolds stress model. This model, named after its developers, use a particular set of equations to close the pressure-strain correlation in the conservation equation of the Reynolds stress tensor.

To gain more detailed information about the turbulence structure in the stator parts, a hybrid large-eddy simulation (RANS-LES) is performed. Additionally, in order to reduce mesh size and computational effort, a wall-modelled, stress-blended eddy simulation (SBES) approach is applied. In this approach, turbulent wall-bounded flow is still modelled using the SST model. The SBES formulation uses a shielding mode to explicitly blend in different turbulence model formulations in RANS and LES modes [16]. The general formulation for the turbulence stress tensor is:

$$
\tau_{i i}^{S B E S}=f_{S} \cdot \tau_{i i}^{R A N S}+\left(1-f_{S}\right) \cdot \tau_{i i}^{L E S} .
$$

The computational mesh for SBES is created according to Menter`s recommendations [16] using:

$$
N_{x} \approx \frac{\delta}{\Delta x} \approx 10 ; \quad N_{y}=30-40 ; \quad N_{z} \approx \frac{\delta}{\Delta z} \approx 20 .
$$

The computational domain, shown in Fig. 2, comprises just one single return channel blade passage with a portion of the radial diffusor and return bend of the same pitch upstream, and finally the $90^{\circ}$-bend with the same pitch downstream. Because the flow conditions at the exit of the $90^{\circ}$-bend might not be very homogenized, an annular, axial domain is added at the $90^{\circ}$-bend exit to avoid falsifying the CFD results through the impact of the outlet boundary conditions. The mesh size for the RANS calculation is determined to be 3 million cells by performing a convergence study. The averaged dimensionless wall distance is $\overline{y^{+}}<1$. The SBES mesh instead comprises 30 million cells. It is generated based on Menter's guideline [16]. The extra axial domain mentioned above is also used in the SBES, but its mesh resolution is chosen to be similarly to that used in the RANS calculation.

Inlet boundary conditions for spanwise total pressure, total temperature and flow angle profiles are taken from experimental data. The flow data is measured at 4 circumferential positions with 12 spanwise positions each. The measured profiles are area-averaged in circumferential direction and a curve fit is applied to obtain boundary conditions for CFD. Measured mass flow rate is set as the outlet condition.

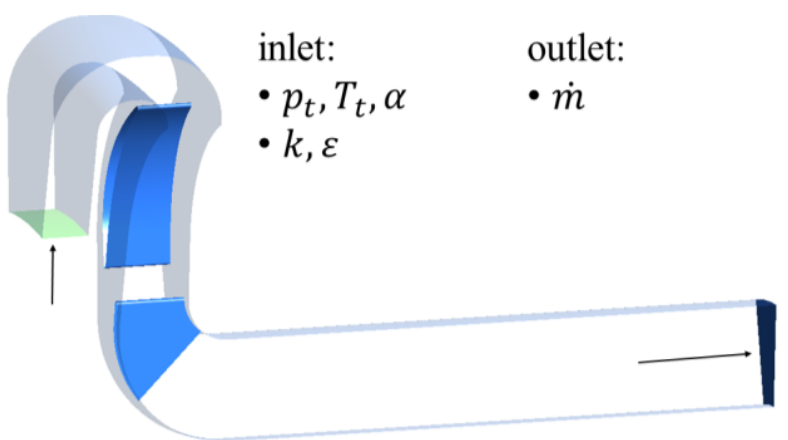

Figure 2: Sketch of the CFD domain

Turbulence inlet conditions obtained from hot-film anemometry (HFA) are applied as spanwise profiles of turbulent kinetic energy $k$ and the turbulent dissipation rate $\varepsilon$. These values are calculated from the measured time series of the velocity according to Bruun [17]:

$$
k=\frac{\overline{u^{\prime}(t)^{2}}}{2}
$$

and

$$
\varepsilon=15 v \frac{\overline{u^{\prime}(t)^{2}}}{\lambda^{2}}
$$

with the integral length scale

$$
\lambda=\bar{u}\left[\overline{\overline{\left(\partial u^{\prime} / \partial t\right)^{2}}}\right]^{1 / 2}
$$

and the velocity

$$
\boldsymbol{u}(\boldsymbol{t})=\overline{\boldsymbol{u}}+\boldsymbol{u}^{\prime}(\boldsymbol{t})
$$

The resulting turbulent inlet profiles for design point conditions are shown in Fig. 3. Centrifugal forces in the impeller (Franz [18]) transport highly energetic fluid toward the hub wall, which results in a typical weak flow at the shroud wall. This results in shear layer at around $50 \%-95 \%$ channel height. Thus, turbulent kinetic energy $\mathrm{k}$ increases with channel height from hub to shroud. The dissipation rate $\varepsilon$ is high between the maxima of $k$, at the shroud wall, and the maximum velocity, at the hub wall. That clarifies the transport character of turbulence. Because macroscopic eddies, generated near shroud wall move toward hub and dissipate. Calculated values by CFD are much higher and thus not comparable to 
measured values of $\varepsilon$, because even thin hot-film probes are much larger than smallest dissipative structures and thus not able to resolve them in space. For this reason, only numerical values of the diffusion rate are discussed. But it is found by the authors, that the shape of the dissipation rate profile at domain inlet is more influential than the absolute level.

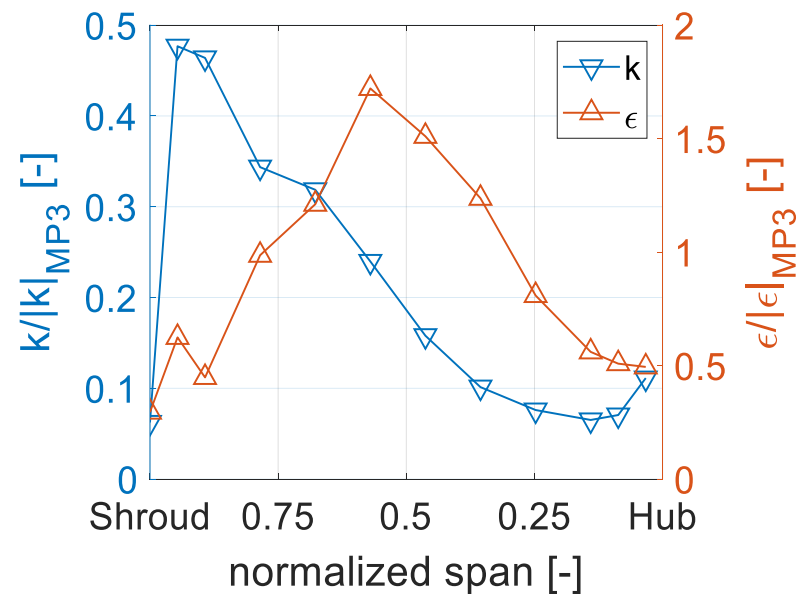

Figure 3: Experimental turbulent spanwise inlet profiles $\boldsymbol{k}$ and $\varepsilon$, area-averaged in circumferential direction

Since an LES domain resolves turbulent eddies, the statistical boundary conditions need to be converted into velocity fluctuations. Therefore, a harmonic flow generator (HFG) based on the work of Kraichnan [19], Smirnov [20] and Batten [21] is implemented in CFX. The HFG derives a turbulent length and time measure from $k$ and $\varepsilon$ to calculate a velocity fluctuation using a Fourier series.

\section{RESULTS}

The return channel performance in terms of total pressure loss and pressure recovery is illustrated in Fig. 4 and 5. In general, the global performance predicted by the SST model fits well with the experimental data. Both the SBES and the SSG model exhibit higher losses and lower pressure recovery.

All numerical solutions consistently show a near linear pressure loss along the return channel. In the diffusor (MP2-MP3), experimental results show a slightly higher pressure loss compared to the CFD results which is caused by mixing of secondary flows induced by the preswirler. Nevertheless, the CFD results indicate that delaying mixing and subsequent excessive turbulence lead to increased losses, especially in the vaned return channel, as will be shown below.

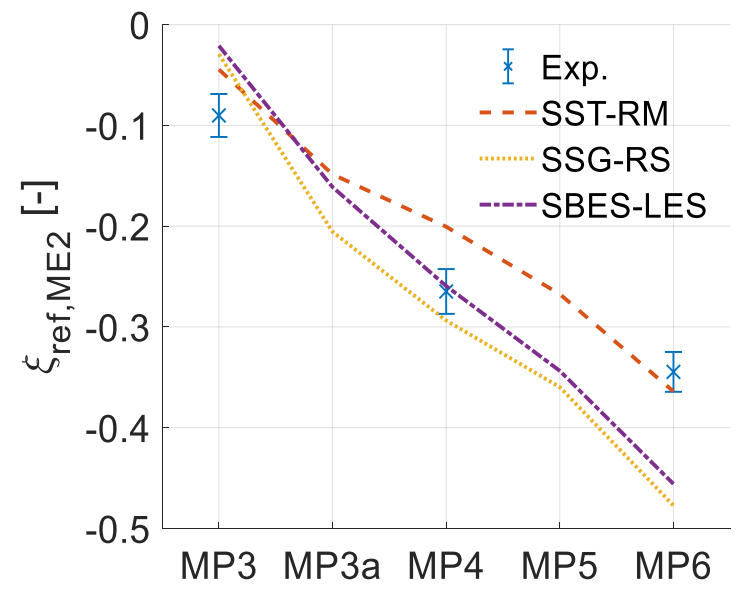

Figure 4: Total pressure loss coefficient referenced to diffuser inlet (MP2)

As with the pressure losses, the SST model is superior to other CFD calculations and agrees very well with the experimentally-determined pressure recovery data, while both the SSG model and SBES predict a distinctly lower recovery, partially caused by higher pressure losses.

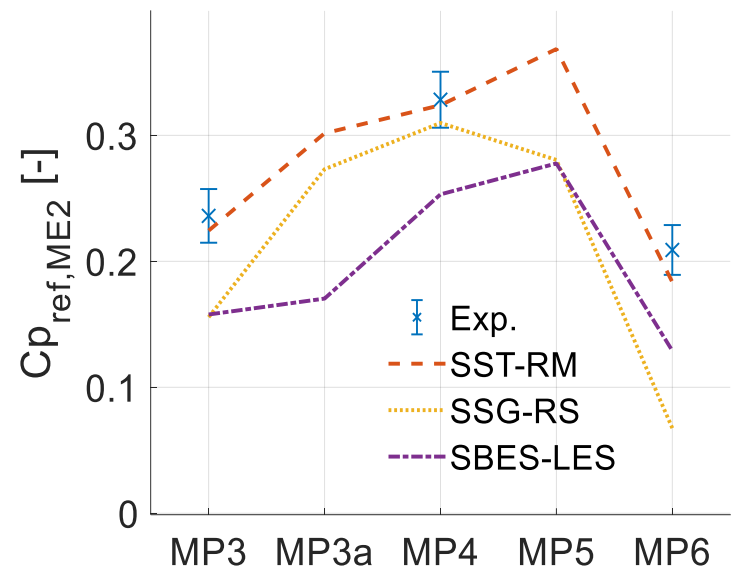

\section{Figure 5: Pressure recovery coefficient referenced to diffuser inlet (MP2)}

Turning to the flow angle $\alpha$, at the inlet of the U-turn, in Fig. 6, the numerical data is in good agreement with measured angles between the hub and $70 \%$ relative channel height. However, at the shroud wall, only the SST results offer a close match, with both other solutions differing; in particular, SBES gives a strong negative flow angle. However, SBES solutions to the Mach number profiles (Fig. 6) indicate a very low velocity near the shroud wall, implying that already minor negative radial velocities lead to high negative flow angles. Nevertheless, the SSG model and SBES estimate a higher separationinduced blockage compared to both the SST model and experimental data, which results in a higher velocity at the hub wall. 
A noticeable Mach number increase at around 20\% channel height is given by every numerical approach. This might be an effect of underestimating turbulent mixing in the diffuser. A slightly hub-sided acceleration is plausible as it is induced by the upstream potential field of the return bend. However, the experimental data show a much more homogeneous flow profile caused by mixing processes. Small differences in the averaged Mach number are caused by the variation of pressure losses in the diffuser.
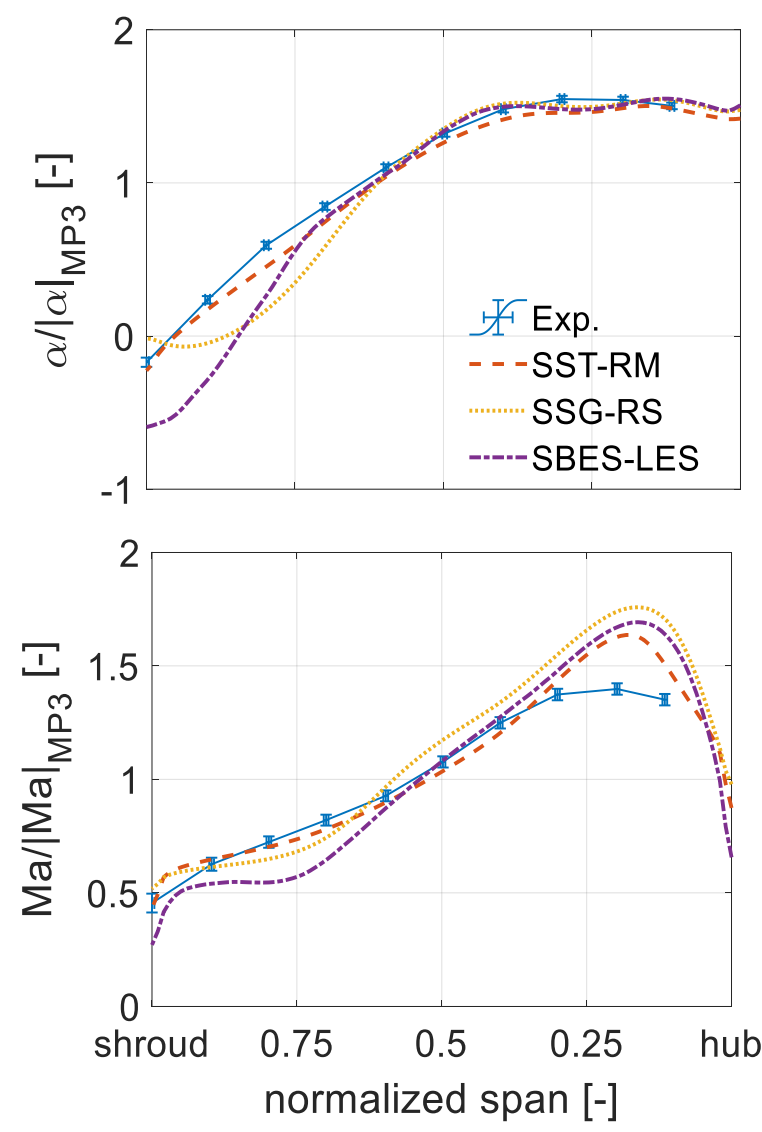

Figure 6: Spanwise and normalized profiles of flow angle $\alpha$ (top) and Mach-number (bottom) in MP3

The corresponding turbulence profiles of turbulent kinetic energy $k$ and dissipation rate $\varepsilon$ are shown in Fig. 7. The SBES and RANS approaches differ in their profiles of $k$, although all are lower than the measured profiles.

The kinetic energy of the SBES, which is the trace of the Reynolds stress tensor, along with the SST and SSG models give their highest values at around 60\% span, although the maximum value given by SSG model is $30 \%$ lower. The difference in energy levels between 10 and $40 \%$ span is also notable. The SST and SSG models both predict a strong decrease of $k$ where the velocity gradient vanishes at the peak of the Mach number (see Fig. 5), whereas SBES predicts a wide plateau at much higher level. This accords with the results by Kim and Chen [13] indicating that turbulent energy can be high at flow regimes where velocity gradients vanish. Whereas, turbulent kinetic energy, in general produced in shear layers is transported lateral via convection and diffusion and finally dissipated.
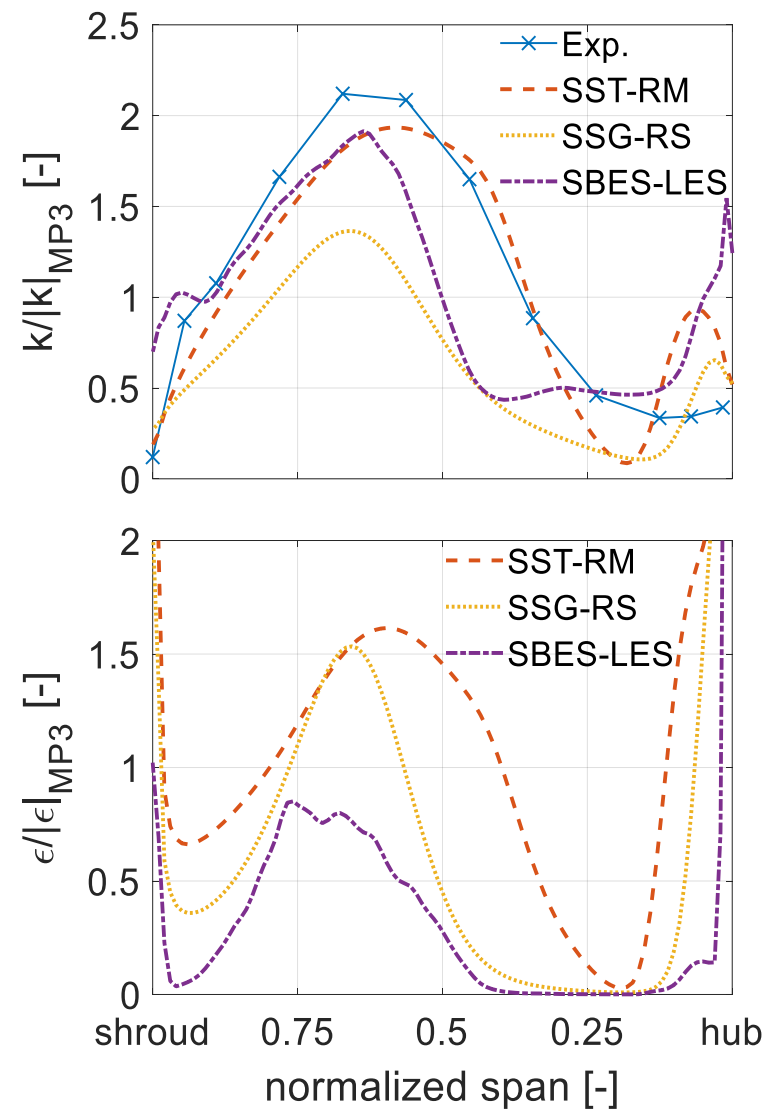

Figure 7: Spanwise and normalized profiles of turbulent kinetic energy $\boldsymbol{k}$ (top) and dissipation rate $\varepsilon$ (bottom) in MP3

The high local level of $k$ in conjunction with an overall low level of dissipation (see Fig. 7) facilitates better mixing processes in the SBES than in the RANS simulations, whereas the higher level of $\varepsilon$ in the SST and SSG simulations suppresses the transport and forces a quicker reduction of $k$ and thus reduces the diffusion and convection of momentum lateral to meridional flow.

In consequence, the SBES flow profile after the first half of the return bend (MP3a) becomes slightly more homogeneous. In particular, at the shroud wall, where $k$ in MP3 was higher and $\varepsilon$ lower compared to RANS, the flow angle $\alpha$ (Fig. 8) becomes not only positive, but distinctly larger. Likewise, the Mach number obtained from SBES increases at the shroud wall and corresponds to the measurements quite well above the $80 \%$ span. However, between $35-80 \%$ span, SBES underpredicts the Mach number along with the RANS results. Thus, from the development in flow profiles from station MP3 to MP3a, it appears that the mixing process is higher in SBES compared to RANS, but nonetheless the calculated profiles still fail to predict the measured ones. 

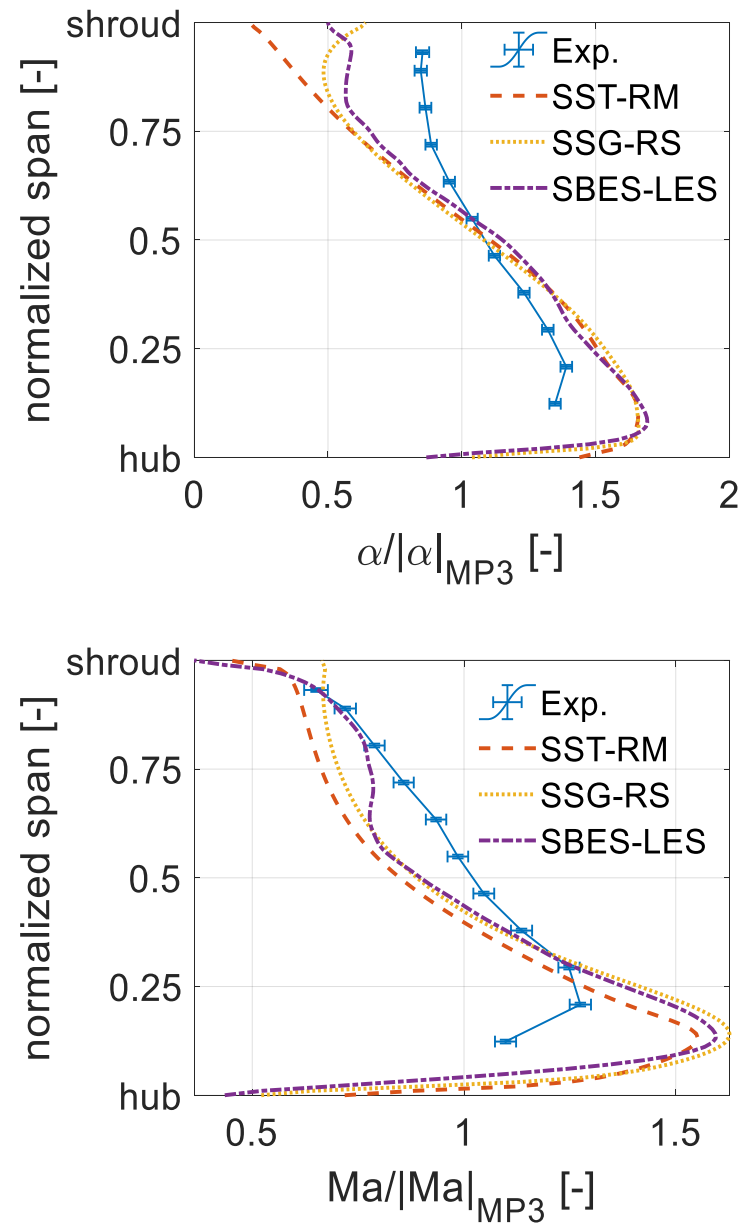

Figure 8: Spanwise and normalized profiles of $\alpha$ (top) and Ma (bottom) in MP3a

The local turbulence profiles of $k$ and $\varepsilon$ in (Fig. 9) imply a further accelerated homogenization of the SBES flow profiles. In this model, turbulent fluctuations increase in such a way that turbulent kinetic energy level markedly exceeds those predicted by the RANS models along the complete channel height. Furthermore, the SBES $k$-profile exhibits a sudden increase near the hub wall, which accords with the experimental data. This $k$ peak supports the procedure of lateral transport of highenergetic fluid toward the shroud wall. Due to advanced homogenization shown by experimental data, the corresponding measured turbulent kinetic energy is lower compared to CFD results. Especially, around mid-span, numerical velocity profiles show a strong gradient leading to high $k$-values. Measured values of $k$ increase only at the walls. The dissipation rate in the SBES increases within the first half of the bend; however, the $\varepsilon$-profile remains lower than in RANS although the gap between RANS and SBES shrinks.
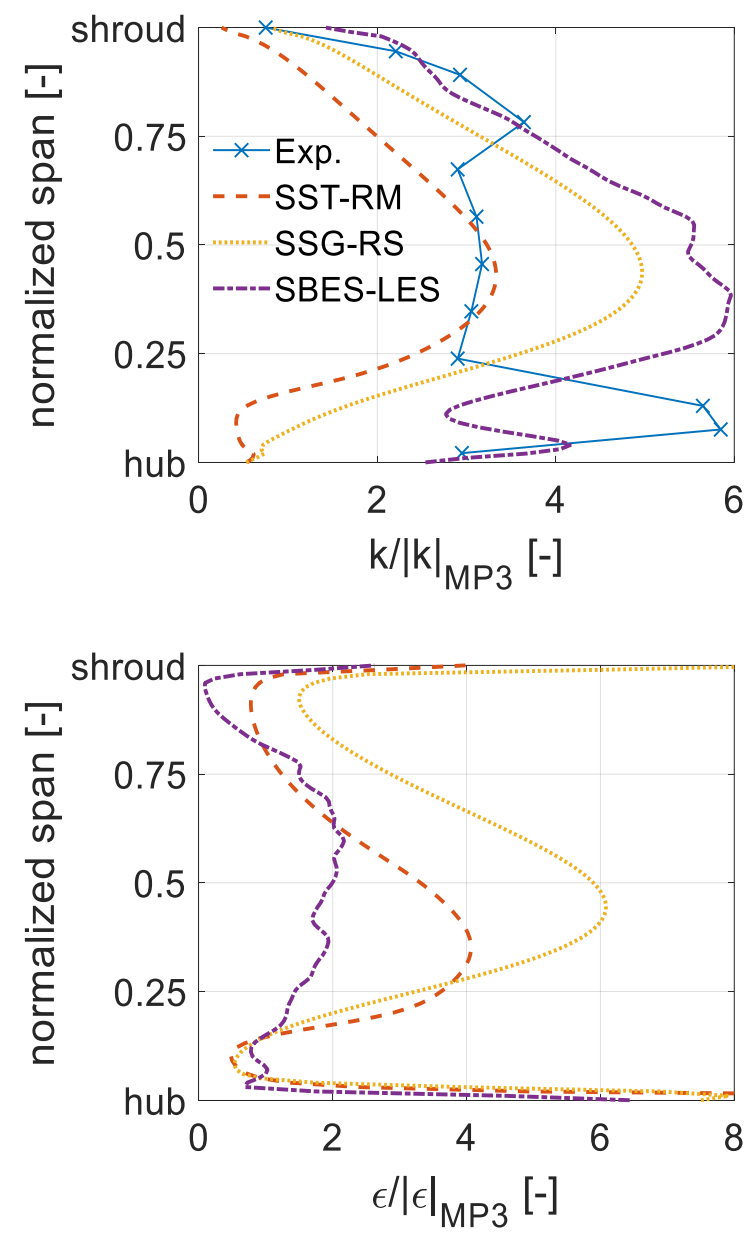

Figure 9: Spanwise and normalized profiles of $\boldsymbol{k}$ (top) and $\varepsilon$ (bottom) in MP3a

This distinct increase in turbulence results in a smooth flow profile at the return bend outlet, MP4. The SBES and experimental profiles of flow angle $\alpha$, depicted in Fig. 10, are in excellent agreement. Unfortunately, in contrast, the SST model and even the SSG model still predict a pronounced variation in flow angle along the channel height. The strong convex curvature of the hub wall in the bend weakens the local wall-bounded flow. As a consequence, the hub-side flow angle rapidly decreases. Additionally, dominant turbulent mixing in the SSG and especially in the SBES simulation cause a further weakening of the hub sided flow. This results in smaller meridional velocity and thus flow angles and more pronounced turbulence.

On the other hand, convex curved walls were found to stabilize turbulent boundary layers according to Muck et al. [22] and preclude flow separation in MP4.

The profiles of turbulent quantities $k$ and $\varepsilon$ in MP4 are given in Fig. 11. The level of every turbulent energy profile, both measured and calculated, decreases from MP3a to MP4. In particular, the profile produced by SBES becomes very broad and flat compared to those produced by the RANS models. This emphasizes the turbulent transport mechanism because the SBES profile 
of $k$ plateaus toward hub wall, with minimal gradient. The highly energetic eddies, generated by instabilities in a shear layer at the channel center at MP3a, move towards the hub and shroud wall and induce the obvious homogenization. This process is less pronounced in both RANS profiles as indicated by the sharper profiles of the turbulent energy together with its lower levels. Furthermore, the level and shape of the corresponding dissipation rate profiles remain similar to those at MP3a but with a little decrease.

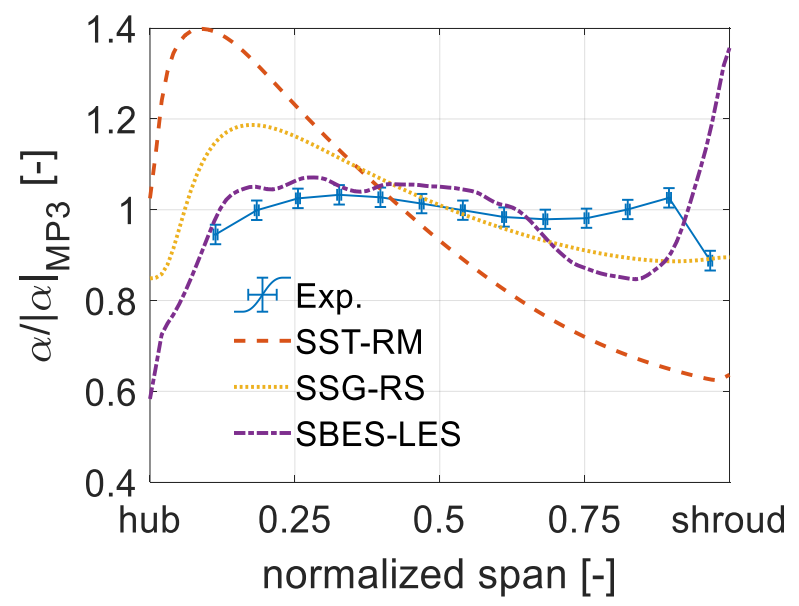

\section{Figure 10: Spanwise and normalized profiles of $\alpha$ in MP4}

Unfortunately, no probe data from MP5 (return guide vane exit) is available yet as space for traversing is very limited. Thus, only, numerical data of turbulent kinetic energy is shown in figure 12 . In the case of virtually undisturbed inflow to the vaned return passage, as calculated by SBES and experimentally confirmed, free shear layers only result from secondary flows such as passage or horseshoe vortices. The resulting shear rates are almost negligible compared to those generated by velocity gradients in the main flow in the diffuser and the return bend. This accounts for the string decrease in turbulent kinetic energy along the vane passage (MP4MP5). The resulting diminished kinetic energy profiles in MP5 can be seen in Fig. 12. The dissipation of the high turbulent energy level in the vaned channel is a main reason why the numerically calculated pressure losses across the channel are higher than the measured losses (see figure 4). The dissipation of turbulent energy in the SST model is lower, thus the pressure loss is lower as well and is in better agreement with the experimental data. An additional dissipation of $\mathrm{k}$ across the subsequent $90^{\circ}$-bend results in further losses.
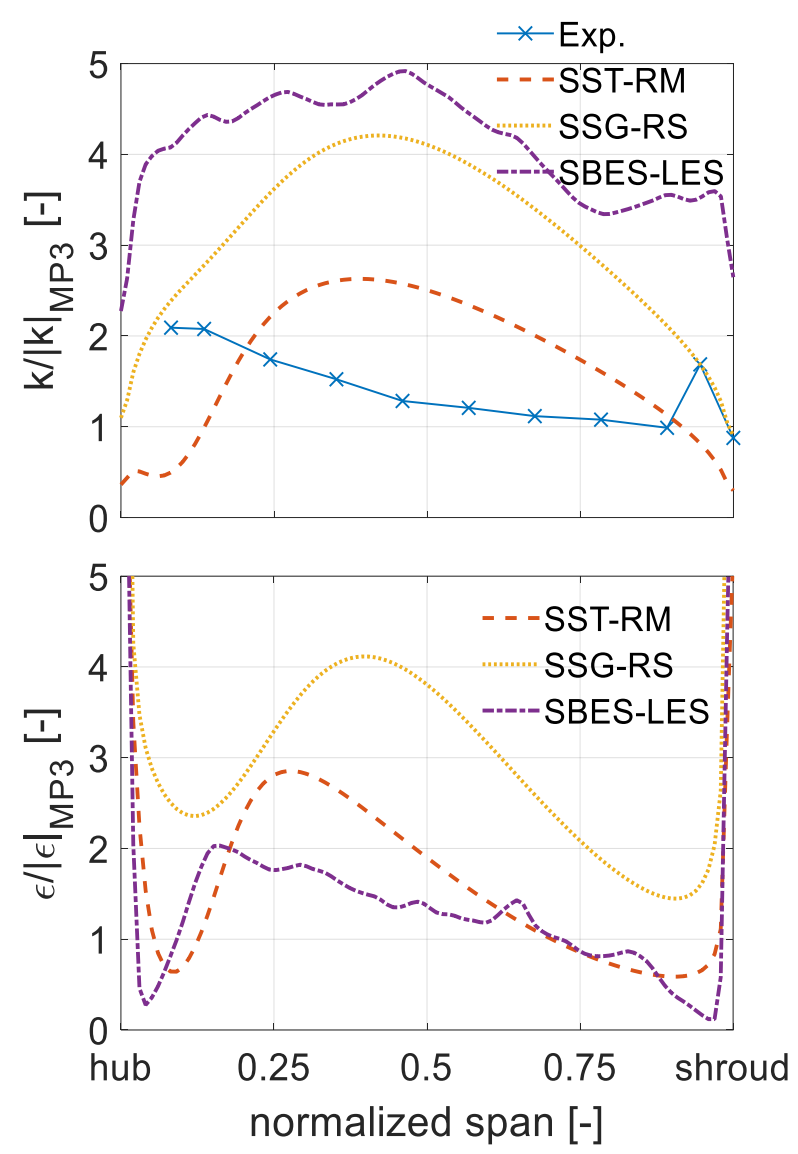

Figure 11: Spanwise and normalized profiles of $k$ (top) and $\varepsilon$ (bottom) in MP4

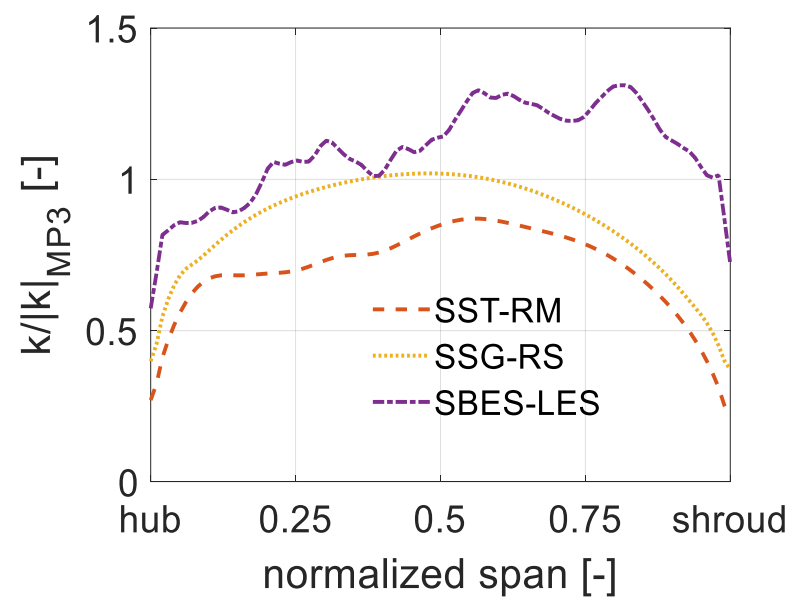

Figure 12: Spanwise and normalized profiles of $k$ in MP5

Figure 13 illustrates the profiles of the normalized tangential velocity in MP6. All profiles, experimental and numerical, exhibit the same trend. At between $20 \%$ and $60 \%$ relative channel height, the reduction of the tangential velocity component induced by the passage vortex in the upstream vane channel is insufficient. In contrast, wall-bounded, pressure driven flow from pressure- to suction-side in the vane passage generates 
counter-rotating swirl which becomes obvious through the negative tangential velocity at both walls. This overturning, especially at the shroud wall, weakens the tip flow in any subsequent impeller and contributes to flow separation.

In comparison to the measured data, the SST model overpredicts the remaining tangential velocity near the mid-span but predicts a lower overturning at the shroud wall. The SBES results show a similar deviation from the measurements. Finally, the SSG model exhibits less span-wise variation and, notably, the negative tangential velocity at the shroud side is lower than the measured data, and here the Mach number profiles become very homogeneous. The best fit to experimental data is provided by SBES, although the profile is slightly higher due to minor pressure recovery. The decline of the Mach number seen in both cases near the shroud wall is typically of turbulent bend flows. In contrast, both RANS models predict a linear decline which accords better with minor mixing. The observed difference is a further indication of the deficits of the RANS models in predicting turbulent flows.
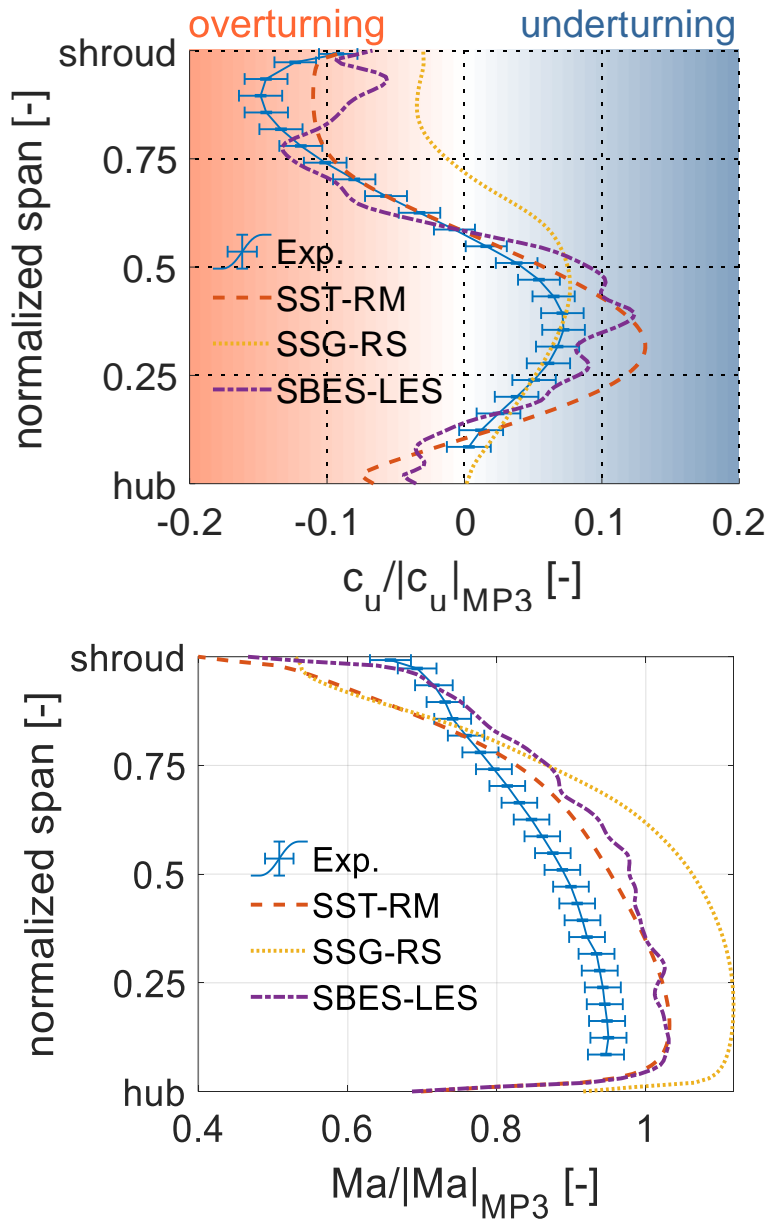

Figure 13: Spanwise and normalized profiles of tangential velocity $c_{u}$ (top) and $\mathrm{Ma}$ (bottom) in MP6

This decline in the linear velocity is surprising, because the concomitant turbulent kinetic energy $\mathrm{k}$ near the shroud predicted by the RANS models exceeds both that determined experimentally and that predicted by SBES (Fig. 14). However, the dissipation rate is more than ten times as high as that calculated by SBES and results in suppressed turbulent mixing.
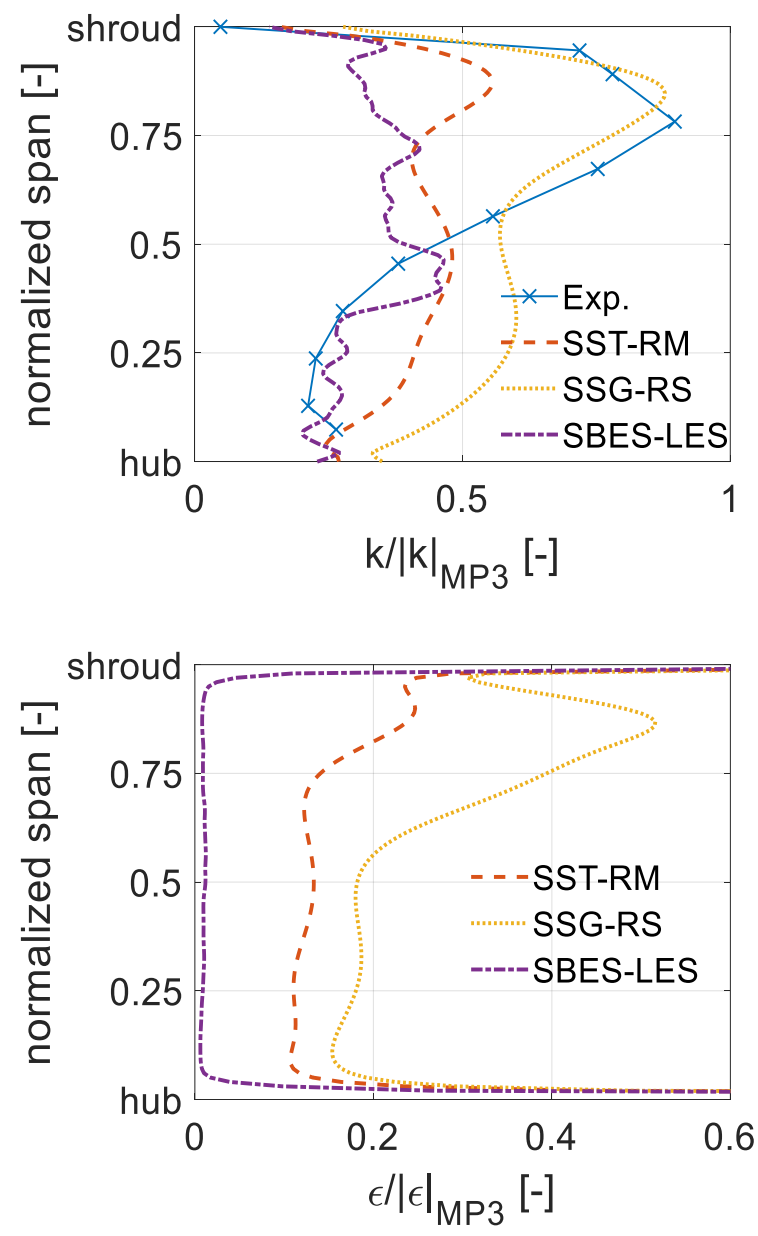

Figure 14: Spanwise and normalized profiles of $\boldsymbol{k}$ (top) and $\varepsilon$ (bottom) in MP4

\section{CONCLUSION}

The comparison of experimental and numerical results demonstrates that RANS simulations exhibits deficits in predicting flow profiles in high flow coefficient return channels of centrifugal compressors. The turbulence profiles show that RANS models calculate either values of turbulent kinetic energy $k$ which are too small, or dissipation rates $\varepsilon$ which are too high. Both deviations result in under-predictions for the turbulent transport lateral to meridional flow direction. This effect is expected to increase with reduced diffuser ratios and discourages a confident geometry design based on numerical results.

Numerical simulations performed using the hybrid LES/RANS approach show that resolved eddies in the LES region (outside the wall boundary) generate a lateral energy and momentum transport in the return- and the $90^{\circ}$-bend sufficient to match the experimental results 
significantly better at the bend outlet. The fact that the flow profiles of the SBES in the diffusor show the same qualitative shape as those obtained from RANS calculations indicate that the applied harmonic flow generator does not transfer enough energy. An increase of inlet turbulent kinetic energy might speed up the turbulent mixing. Since the development of turbulent structures requires time and flow path, the turbulence level in the diffusor is only similar to those given by the RANS calculations, if the boundary conditions for the SBES are insufficient. Only in the return bend does the flow reach a turbulence level which matches experimental data upstream of the vaned return channel. A consequence of turbulent mixing being delayed, and therefore inflow to the return bend being disturbed, is that the resultant turbulence in the bend might be excessive. This is confirmed by the measured turbulent kinetic energy $k$, which is much lower in the bend than that calculated by SBES.

Nevertheless, SBES is an advanced simulation technique that enables better understanding of turbulent effects and the general flow in a return channel. However, only RANS models allow currently economic flow calculations and therefore need to be improved.

\section{NOMENCLATURE}

$\begin{array}{ll}\alpha & \left.\text { flow angle from tangential [ }{ }^{\circ}\right] \\ \boldsymbol{C}_{\boldsymbol{p}} & \text { pressure recovery coefficient [-] } \\ \delta & \text { boundary layer thickness }[\mathrm{m}] \\ \varepsilon & \text { turbulent dissipation rate }\left[\mathrm{m}^{2} / \mathrm{s}^{3}\right] \\ \zeta & \text { pressure loss coefficient }[-] \\ H F A & \text { hot-film-anemometry } \\ k & \text { turbulent kinetic energy }\left[\mathrm{m}^{2} / \mathrm{s}^{2}\right] \\ \lambda & \text { integral length scale }[\mathrm{m}] \\ M a & \text { Mach number [-] } \\ \boldsymbol{N}_{\boldsymbol{x}}, \boldsymbol{N}_{\boldsymbol{y}}, \boldsymbol{N}_{\boldsymbol{z}} & \text { dimensional cell count }[-] \\ v & \text { kinematic viscosity }\left[\mathrm{m}^{2} / \mathrm{s}\right] \\ \tau_{i j} & \text { Reynolds stress tensor }[\mathrm{kg} /(\mathrm{m} \cdot \mathrm{s})] \\ p & \text { static pressure [Pa] } \\ p_{t} & \text { stagnation/total pressure }[\mathrm{Pa}] \\ R G V & \text { return guide vane } \\ S B E S & \text { shield-blended-eddy-simulation } \\ t & \text { time [s] } \\ u & \text { absolute velocity }[\mathrm{m} / \mathrm{s}] \\ y^{+} & \text {dimensionless wall distance }[-] \\ x, y, z & \text { dimensional distance }[\mathrm{m}]\end{array}$

\section{Sub-/Superscripts}

$\begin{array}{ll}\text { in } & \text { inlet } \\ n, M P 2 & \text { normalized quantity; by mean values } \\ & \text { in MP2 } \\ \text { out } & \text { Outlet } \\ - & \text { mean value } \\ & \text { fluctuation }\end{array}$

\section{ACKNOWLEDGMENTS}

This project is funded by BMWi (German Bundesministerium für Wirtschaft und Energie) under contract number 03ET7041F as a part of the joint research program COOREFLEX-Turbo in the scope of AG Turbo. The authors gratefully acknowledge Siemens AG for their support and permission to publish this paper

\section{REFERENCES}

[1] Rothstein, E., "Experimentelle und theoretische Untersuchung der Strömungsvorgänge in Rückführkanälen von Radialverdichterstufen, insbesondere solcher mit geringen Kanalbreiten", PhD thesis, RWTH Aachen University. 1984.

[2] Lenke, L.J., „Numerische Simulation der turbulenten Strömung in Rückführkanälen mehrstufiger Radialverdichter", $\mathrm{PhD}$ thesis, University DuisburgEssen. 1999.

[3] Aalburg, C., Simpson, A., Schmitz, M.B., Michelassi, V., Evangelisti, S., Belardini, E., Ballarini,V., "Design and Testing of Multistage Centrifugal Compressors with Small Diffusion Ratios“, Proceedings of ASME Turbo Expo 2008; GT2008-51263.

[4] Rossbach, T., „Sekundärströmungen im Rückführsystem einer Radialverdichterstufe“, $\mathrm{PhD}$ thesis, RWTH Aachen University. 2019.

[5] Rossbach, T., Rube, C., Wedeking, M., Franz, H., Jeschke, P., "Performance Measurements of a Full Stage Centrifugal Process Gas Compressor Test Rig", $11^{\text {th }}$ European Conference on Turbomachinery Fluid Dynamics Thermodynamics; ETC2015-084.

[6] Wilcox, D.C., "Turbulence modeling for CFD,

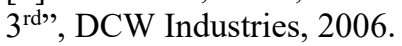

[7] Bisping, J., Rossbach, T., Grates, D., Jeschke ,P., Hildebrandt, A., "Influence Of Diffuser Diameter Ratio On The Performance Of A Return Channel Within A Centrifugal Compressor Stage", Proceedings of GPPS Forum 18; GPPS-2018-0034.

[8] Veress, Á., Van den Braembussche, R., „Inverse Design and Optimization of a Return Channel for a Multistage Centrifugal Compressor", ASME Journal of Turbomachinery 126, 2004.

[9] Aalburg, C., Simpson, A., Carretero, J., Nguyen, T., Michelassi, V., "Extension of the Stator Vane Upstream Across the $180^{\circ}$ Bend for a Multistage Radial Compressor Stage", Proceedings of ASME Turbo Expo 2009; GT2009-59522.

[10] de Bellis, F., Guidotti, E., Rubino, D.T., "Centrifugal Compressors Return Channel Optimization by Means of Advanced 3D CFD", Proceedings of ASME Turbo Expo 2015; GT2015-44143.

[11] Jariwala, V., Larosiliere, L., Hardin, J., "Design Exploration of a Return Channel for Multistage Centrifugal Compressors", Proceedings of ASME Turbo Expo 2016; GT2016-57777.

[12] Dolle, B., Brillert, D., Dohmen, H.J., Benra, F.-K., „Experimental and Numerical Investigation on the Flow in a Return Channel of Multistage Centrifugal 
Compressors", Proceedings of ASME Turbo Expo 2019; GT2019-90455.

[13] Kim, S.W., Chen, Y.S., "A finite element computation of turbulent boundary layer flows with an algebraic stress turbulence model", Computer Methods in Applied Mechanics and Engineering. 1988.

[14] Menter, F.R., "Two-equation eddy-viscosity turbulence models for engineering applications", AIAA Journal 32, 1994.

[15] Speziale, C.G., Sarkar, S., Gatski, T.B., , Modelling the pressure-strain correlation of turbulence: an invariant dynamical systems approach", Journal of Fluid Mechanics 227, 1991.

[16] Menter, F.R., "Best Practice: Scale-Resolving Simulations in ANSYS CFD”, ANSYS Germany GmbH, 2015.

[17] Bruun, H.H., "Hot wire anemometry: Principles and signal analysis", Oxford Univ. Press, 1995.
[18] Franz, H., “ Numerische Untersuchung der Durchströmung einer Rückführung eines mehrstufigen Radialverdichters", PhD thesis, University DuisburgEssen. 2015.

[19] Kraichnan, R.H., "Diffusion by a Random Velocity Field”, Physics of Fluids 13, 2003.

[20] Smirnov, A., Shi, S., Celik, I., "Random Flow Generation Technique for Large Eddy Simulations and Particle-Dynamics Modeling", Journal of Fluid Mechnics 123(2), 2001.

[21] Batten, P., Goldberg, U., Chakravarthy, S., "Interfacing Statistical Turbulence Closures with LargeEddy Simulation", AIAA Journal 42(3), 2004.

[22] Muck, K.C., Hoffmann, P.H., Bradshaw, P., "The effect of convex surface curvature on turbulent boundary layers", Journal of Fluid Mechanics 161, 1985. 